Original

\title{
Molecular biological and histological effects of Er:YAG laser irradiation on tooth movement
}

\author{
Yuji Tsuka1), Ryo Kunimatsu1), Hidemi Gunji1), Kengo Nakajima1), Tomoka Hiraki²), \\ Ayaka Nakatani2), and Kotaro Tanimoto1) \\ 1)Department of Orthodontics, Division of Oral Health and Development, Hiroshima University Hospital, \\ Hiroshima, Japan \\ 2)Department of Orthodontics, Applied Life Sciences, \\ Hiroshima University Institute of Biomedical \& Health Sciences, Hiroshima, Japan
}

(Received December 12, 2017; Accepted February 17, 2018)

\begin{abstract}
The aim of this study was to evaluate the effects of a fiberotomy-like procedure using Er:YAG laser irradiation on the velocity of orthodontic tooth movement. To produce experimental tooth movement in rats, orthodontic force was applied to the upper first molars with a nickel-titanium closed coil. The right molars were irradiated with an Er:YAG laser while the non-irradiated left molars were used as controls. The rats were sacrificed at 4 weeks after the start of tooth movement and the distance between the mesial side of the second molar and the distal side of the upper first molar was measured on CT images. The amount of tooth movement was significantly greater in the irradiation group than in the control group. The TRAP-positive nuclei count at the pressure site was higher in the laser-irradiation group than in the control group. Expression of RANKL and ALP was higher at the mesial-coronal pressure site in the laser-irradiation group than in the control group. In addition, expression of OPG was higher at the pressure site in the control group than in the laser-irradiation group. These results suggest that a fiberotomy-like procedure using an Er:YAG laser
\end{abstract}

Correspondence to Dr. Yuji Tsuka, Department of Orthodontics, Division of Oral Health and Development, Hiroshima University Hospital, 1-2-3 Kasumi, Minami-ku, Hiroshima 734-8553, Japan FAX: +81-82-257-5687 E-mail: tsuka1@hiroshima-u.ac.jp

J-STAGE Advance Publication: February 1, 2019

Color figures can be viewed in the online issue at J-STAGE.

doi.org/10.2334/josnusd.17-0472

DN/JST.JSTAGE/josnusd/17-0472 stimulates osteoclasts and osteoblasts and may promote bone metabolism in the context of experimental tooth movement.

Keywords: Er:YAG laser; tooth movement; fiberotomy; bone metabolism; HLLT.

\section{Introduction}

Orthodontic treatment involves application of force in order to achieve tooth movement, and this requires a period of time. Prolonged orthodontic treatment can cause patient discomfort and is a source of trauma to oral tissue. Therefore, finding methods to minimize the treatment period remains a major challenge for orthodontists. Accelerated experimental tooth movement has been performed using various methods. Surgical procedures include corticotomy $(1,2)$, fiberotomy $(3,4)$, corticision (5), and osteotomy (6). Experimental facilitation of tooth movement using corticotomy has been reported both clinically and in rats; in this situation, tooth movement is increased when an incision is made in the hard cortical bone. Such procedures are relatively invasive. Local injection of agents involved in bone metabolism, such as receptor activator of nuclear factor kappa-B ligand (RANKL), (7,8) prostaglandin E2 (9) and parathyroid hormone (10), has also been used. However, this approach is clinically difficult because of potential side effects. Finally, methods involving the use electromagnetic waves (11), laser treatment $(12,13)$ and vibration (14) are considered to be less invasive and associated with fewer side effects. The possibility of accelerating the move- 


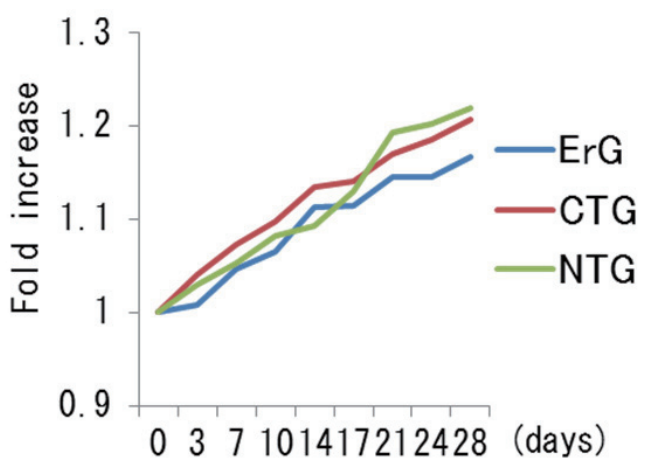

Fig. 1 Photograph and schematic diagram of the apparatus used for tooth movement.

ment of teeth using Nd:YAG laser has been demonstrated so far (15). However, the degree of acceleration was very small. Various studies have shown that both the Nd:YAG laser and Er:YAG laser induce cell activity when used at low power $(16,17)$. Treatments associated with less tissue damage tend to be less efficient at accelerating tooth movement, whereas more invasive treatments such as corticotomy have a greater influence. Aleksic et al. (17) have reported enhanced proliferation of osteoblastlike cells after irradiation with a low-power Er:YAG laser. The Er:YAG laser is a surface absorption-type laser and is capable of cutting teeth when used at high power. Fiberotomy is also reported to accelerate tooth movement (3). Based on these findings, using by Er:YAG laser that fiberotomy-like treatment with was expected to low reactive level laser therapy (LLLT) effect in surrounding tissues. Therefore, it was investigated whether irradiation of Er:YAG laser during experimental tooth movement simultaneously exerts the same effect as fiberotomy and LLLT.

\section{Materials and Methods}

\section{Animals}

The animal experiment protocol used in this study was approved by the Ethics Committee for Animal Experiments (ID A15-43). Eleven male Wistar rats (aged 10 weeks) were used (Charles River Laboratories, Yokohama, Japan). All rats were maintained at the Natural Science Center, and handled in accordance with the ARRIVE guidelines for animal research and the Rules for Animal Experiments. Rats were housed in humidity cages (50-60\%) at the animal center under a 12-h light/dark environment at a constant temperature of $22-24^{\circ} \mathrm{C}$, and provided with food and water. Body weight was measured twice a week.

\section{Experimental tooth movement}

The orthodontic appliance employed was composed of nickel-titanium closed-coil springs (SENTALLOY; Tomy International, Tokyo, Japan) linked to the upper first molars by stainless steel ligature wires ( 0.008 inch; Tomy) stretched to achieve $10 \mathrm{~g}$, and then tied to the upper incisors with secondary ligature wires. Composite resin (Unifil flow, GC, Tokyo, Japan) was used to cover the maxillary incisors and ligature wires (Fig. 1). No gap was created by the wire ligation. In this method, tooth movement was achieved by inclination. The method employed has been established as a tooth movement model $(12,18)$. The duration of tooth movement was 4 weeks. The first maxillary left molar was subjected to laser irradiation, and the right first molar was used as the non-irradiated control group.

\section{Laser irradiation}

An Er:YAG laser (Erwin AdvErL, Morita, Tokyo, Japan) with a wavelength of 2,940 $\mathrm{nm}$ was used in this study. Irradiation was performed for $120 \mathrm{~s}$ at three time points (total of $360 \mathrm{~s}$ ) per day on days $0,1,2,3,7,10,14,17$, 21 , and 24 (total of ten times). Laser irradiation was performed at $30 \mathrm{~mJ}$ and $10 \mathrm{pps}(0.3 \mathrm{~W})$. Total energy exposure was $54 \mathrm{~J}$, which was similar to the dose used in a previous study (18). The laser beam was delivered by a $0.8-\mathrm{mm}$-diameter optical fiber (C800F, Morita) and irradiation was administered by placing the end of the optical fiber tip in contact and surround moved with the mesial, buccal and palatal sides of the upper first molar gingiva.

\section{Measurement of tooth movement by $\mu \mathrm{CT}$}

Computed tomography (CT) images were taken using a $\mu \mathrm{CT}$ machine (Sky Scan 1176; Bruker microCT, Kontich, Belgium). Images were taken in vivo with a pixel size of $35 \mu \mathrm{m}$. CT imaging was carried out every week under general anesthesia after starting tooth movement. The position was defined by the plane perpendicular to the medial palatal suture of the rat and the occlusal plane. The distance between the most prominent portion of the central lobe on each crown's facial surface's on the mesial side of the second molar and the distal side of the upper first molar on $\mathrm{CT}$ images was measured using analysis data viewer software (Bruker microCT). Intergroup comparisons of average values were performed by $t$ test analysis. Resulting $P$-values of less than 0.05 were considered to indicate significant differences.

\section{Tissue preparation}

After 4 weeks, the animals were sacrificed using an 


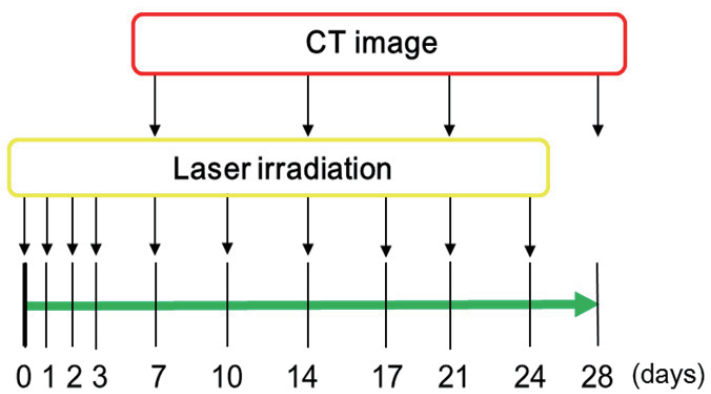

Fig. 2 ErG: tooth movement and Er:YAG Laser irradiation; CTG: tooth movement alone; NTG: no treatment. No differences in whole body weight were observed between the non-treated and control groups.

intraperitoneal injection of sodium pentobarbital (somnopentyl $5 \mathrm{mg} / 100 \mathrm{~g}$; Kyoritsuseiyaku, Tokyo, Japan), and tissues were fixed by perfusing the animals through the ascending aorta with 4\% paraformaldehyde. Specimens were decalcified in 14\% EDTA and embedded in paraffin. Sagittal serial sections $(5 \mu \mathrm{m})$ were prepared using a rotary microtome (Microm325; Carl Zeiss, Oberkochen, Germany), and subjected to hematoxylin-eosin or tartrate-resistant acid phosphatase (TRAP) staining. TRAP staining was performed using a TRAP staining kit (Wako, Tokyo, Japan). Counting of osteoclast nuclei was performed in two separate areas in each selected section. These two areas were located mesio-coronal to the pressure site and disto-apical to the pressure site at the mesial root. The overall size of each measurement area was $1,175 \mu \mathrm{m} \times 815 \mu \mathrm{m}$. Student's $t$ test was performed to determine differences in the localization of TRAP-positive nuclei. Expression levels of RANKL, osteoprotegerin (OPG), and alkaline phosphatase (ALP) were observed by immunohistochemical staining. Briefly, primary antibodies against RANKL (sc-7628, 1:100 dilution; Santa Cruz, Heidelberg, Germany), OPG (sc-11383, 1:50 dilution; Santa Cruz,) and ALP (ab108337, 1:500 dilution; Abcam, Tokyo, Japan) were used with a Vectastain Elite ABC staining system (Vector Labs, Burlingame, CA, USA). Counterstaining was carried out with hematoxylin, and analysis was performed using image analysis software (BZ Analyzer software BZ-H1A; Keyence, Osaka, Japan).

\section{Statistical analysis}

Results are expressed as means \pm standard deviation. The Wilcoxon $t$ test was used for comparisons between two groups, and one-way analysis of variance with Bonferroni post-hoc test was used for comparisons between multiple groups. The significance level was set at $P<$ 0.05 or $<0.01$.
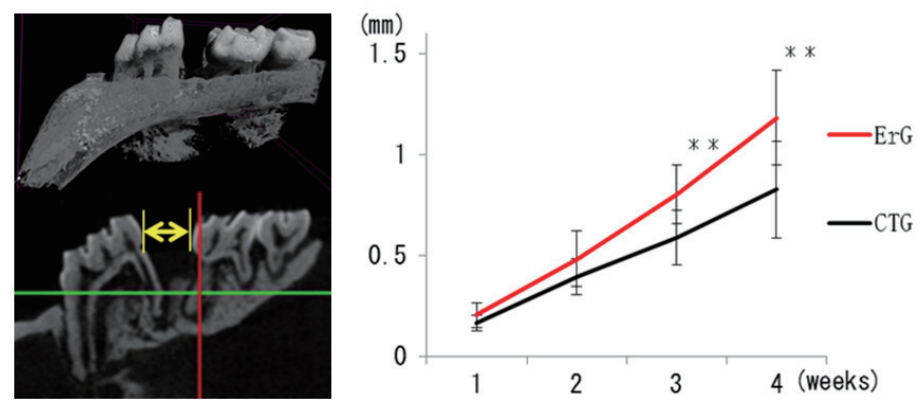

Fig. 3 Measurement of distance between the mesial side of the second molar and the distal side of the upper first molar on the CT image (arrows). As shown in the figure on the right, there was significantly greater movement in the irradiation group after 3 weeks and 4 weeks. ** $P<0.01$ vs. control, $n=11$

\section{Results}

\section{Effects of laser irradiation on body weight}

No differences in whole body weight were observed between the non-treated and control groups, suggesting that use of the orthodontic appliance did not influence feeding. The laser-irradiated rats tended to have a lower body weight than the control group, but the difference was not statistically significant (Fig. 2).

\section{Amount of tooth movement}

The space between the first and second molars was determined (Fig. 3, arrows). The amount of tooth movement was significantly greater in the irradiation group than in the control group after 3 weeks and 4 weeks (Fig. 3).

\section{Histopathological observation TRAP staining}

There was a significantly higher count of TRAP-positive nuclei at the mesio-coronal pressure site in the laserirradiation group than in the control group. No significant differences in the count of TRAP-positive nuclei were observed at the disto-apical pressure site (Fig. 4).

\section{HE staining \& IHC staining}

Teeth showed movement as a result of crown inclination in all groups. Irregularities in periodontal ligament fibers and absorption of alveolar bone were observed at the pressure sites in the mesio-coronal and disto-apical areas, regardless of laser irradiation (Figs. 5, $6 \mathrm{HE}$ ). Expression of RANKL was higher at the mesio-coronal pressure site in the laser-irradiation group than in the control group (Figs. 5, 6 RANKL).

Expression of OPG was lower at the mesio-coronal pressure site in the laser-irradiation group than in the control group (Figs. 7, 8 OPG).

Expression of ALP was higher at both the mesiocoronal pressure site and the disto-apical pressure site 

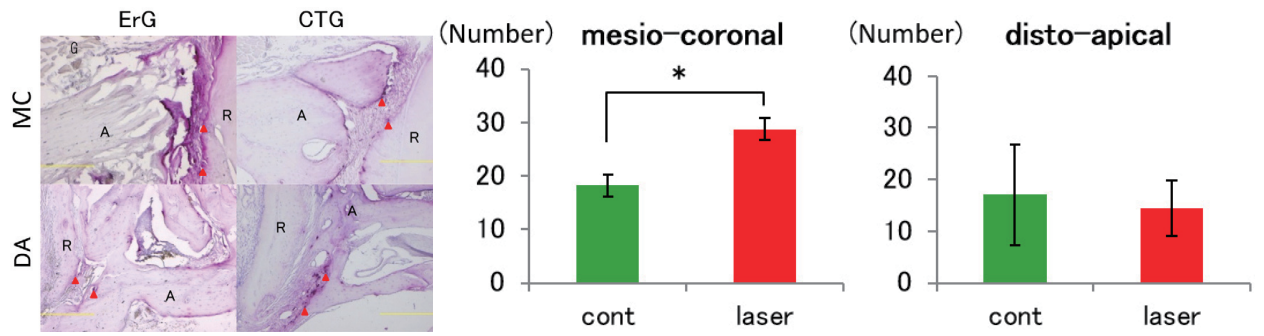

Fig. 4 MC: mesio-coronal; DC: disto-apical. There was a significantly higher count of TRAPpositive nuclei in the mesio-coronal pressure zones in the laser-irradiation group than in the control group.

$* P<0.05$

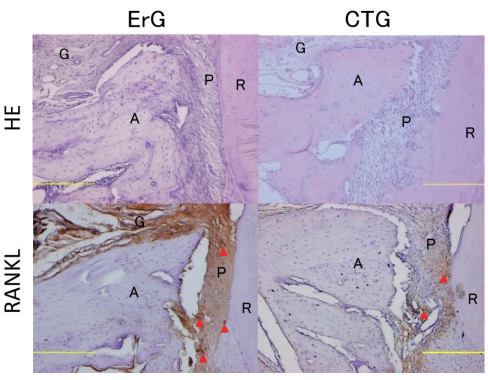

Fig. 5 Mesio-coronal side.

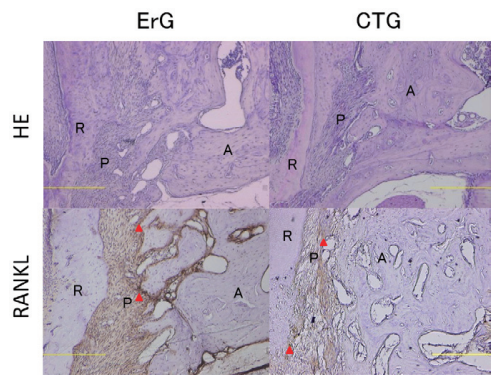

Fig. 6 Disto-apical side.

G: gingiva; A: alveolar bone; R: root; P: periodontal tissue; HE staining: in the control group, irregularities of the endodontic ligament fibers and alveolar bone resorption were observed in compression areas in the mesio-coronal and disto-apical regions; RANKL staining: expression of RANKL was higher in the mesio-coronal pressure zones in the laser-irradiation group than in the control group.

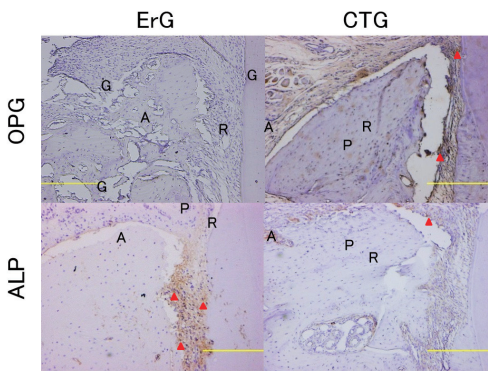

Fig. 7 Mesio-coronal side.

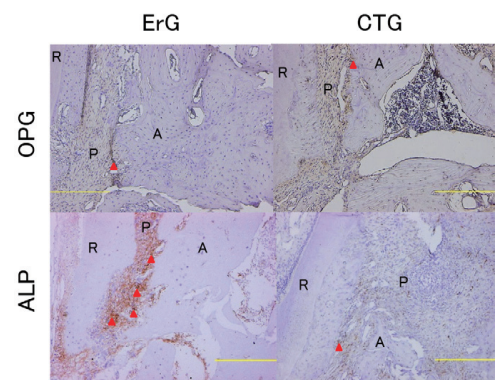

Fig. 8 Disto-apical side.

OPG staining: expression of OPG was lower in the mesio-coronal pressure zones in the laserirradiation group than in the control group. ALP staining: expression of ALP was higher in the mesio-coronal pressure zones in the laser-irradiation group than in the control group.

in the laser-irradiation group than in the control group (Figs. 7, 8 ALP).

\section{Discussion}

The total area of the upper first permanent molar in humans is reported to be $340 \mathrm{~mm}^{2}$, which is 32 times that of the rat. Accordingly, a force of $10 \times \mathrm{g}$ used on a rat molar would correspond to $340 \times \mathrm{g}$ on a human molar (19). According to the differential force theory proposed by Begg, the optimal force value needed to move a human molar is $300-500 \mathrm{~g}(20)$. Therefore, in this study, it was decided to use 4 weeks the Ni-Ti closed coil to apply a force of $10 \mathrm{~g}$. The Er:YAG laser supplies far-infrared radiation from a mid-infrared laser, and the wavelength of the pulse wave oscillation is $2,940 \mathrm{~nm}$. In the present study, the effects of the Er:YAG laser on tooth movement were investigated metrically and histologically. It is known that pulsed lasers exert effects other than mechanical stimulation (21). Er:YAG lasers do not reach deep tissue and do not directly affect the tooth root. However, it is possible that the reaction on the periodontal tissue surface indirectly affects deep areas such as the 
root. Fujita et al. (22) indicated that the amount of tooth movement on day 7 was significantly greater in a laserirradiation group than in a non-irradiation group. Another study has also shown that the amount of tooth movement on day 7 was significantly greater in an irradiation group than in a control group (15). However, there was no difference in the amount of tooth movement on days 14, 21, and 28. It was found that the Nd:YAG laser only affects early tooth movement. In the present study, the amount of tooth movement was significantly greater (1.4-fold) in the irradiation group after 3 weeks and 4 weeks than in the control group. At 4 weeks after the start of pressure application, greater tooth movement was evident in the Er:YAG laser-irradiation group than in the Nd:YAG laser-irradiation group. One reason for this may have been the difference in the mechanism of action. In a study where fiberotomy was performed during experimental tooth movement, Tuncay et al. reported that the amount of tooth movement was 1.2 times that in the untreated control group. The Er:YAG laser acts primarily on the tissue surface. Very little surface bleeding was observed after Er:YAG laser irradiation in the present study. The condition of the tissue after Er:YAG laser irradiation under the present conditions may be similar to that after incision of the fibers. The Er:YAG laser generates a range of temperatures in the target tissue, destroying or altering its architecture. This is referred to as high reactive level laser treatment (HLLT). Simultaneously, non-destructive thermal and non-thermal bioactivation occur at the periphery of the target tissue; this is referred to as "simultaneous LLLT" and can explain some of the advantages of laser irradiation (16). In other words, HLLT occurs at the tissue surface, and LLLT occurs in the surrounding areas. HLLT is known to produce a photobiodestructive reaction resulting in cellular vaporization, whereas LLLT generates a photobioactive reaction (PAR) to stimulate cellular proliferation and differentiation (23). In this study, it is considered that mild destruction and HLLT occurred in the tissue after irradiation with Er: YAG laser. In this study, a greater degree of tooth movement was observed than that reported by Tuncay et al. This may have been due to the fiberotomy-like effects and additive effects of HLLT and LLLT after irradiation.

The count of TRAP-positive nuclei was also significantly higher at the mesio-coronal pressure site after laser irradiation when compared to the control group (Fig. 4). In addition, the expression of RANKL was greater at the mesio-coronal pressure site in the laser-irradiation group than in the control group. These findings are consistent and suggest that bone resorption was increased. Furthermore, expression of ALP was higher at the mesio- coronal pressure site in the laser-irradiation group than in the control group. It is possible that laser irradiation promotes bone remodeling, involving both bone resorption and bone regeneration. Further studies are required to examine the clinical applications of the Er:YAG laser. Ninomiya et al. (21) reported that pulsed laser irradiation increased bone volume and the mineral apposition rate. In addition, increasing the number of pulses was effective for bone formation. They also observed that laser irradiation enhanced expression of ALP and was similar to the results of this study. Ogasawara et al. (24) examined the expression of RANKL in osteoblasts and periodontal ligament cells during orthodontic tooth movement and emphasized the importance of RANKL-OPG release and their interaction in bone remodeling. In the present study, higher RANKL expression was observed on the laser irradiation side, whereas OPG expression was decreased. Thus, a higher RANKL/OPG ratio would have been expected, and this might have contributed to tooth movement acceleration. There were no significant differences in the count of TRAP-positive nuclei at the disto-apical pressure site in either the laser-irradiated group or the control group. This may have been due to the dynamic pattern of tooth movement, which crown is tilting movement. Pressure on the distal side would have decreased as tooth movement advanced.

This present method is advantageous because it is less invasive than corticotomy. In addition, due to the effects of HLLT, healing may be faster than that after conventional fiberotomy, and may accelerate tooth movement to a greater degree than with LLLT.

In view of the above considerations, a new method for accelerating the orthodontic movement of teeth, using Er:YAG laser, proposes such fiberotomy-like procedure. The present results also suggest that laser irradiation stimulates osteoclasts and osteoblasts, thereby promoting bone metabolism during tooth movement.

\section{Acknowledgments}

This study was funded by JSPS KAKENHI Grant Number 16K20644 from the Ministry of Education, Culture, Sports, Science and Technology of Japan.

\section{Conflict of interest}

The authors have no potential conflict of interest with respect to the authorship and/or publication of this article.

\section{References}

1. Kole H (1959) Surgical operations on the alveolar ridge to correct occlusal abnormalities. Oral Surg Oral Med Oral Pathol 12, 515-529. 
2. Al-Naoum F, Hajeer MY, Al-Jundi A (2014) Does alveolar corticotomy accelerate orthodontic tooth movement when retracting upper canines? A split-mouth design randomized controlled trial. J Oral Maxillofac Surg 72, 1880-1889.

3. Tuncay OC, DM Killiany (1986) The effect of gingival fiberotomy on the rate of tooth movement. Am J Orthod 89, 212-215.

4. Young L, Binderman I, Yaffe A, Beni L, Vardimon AD (2013) Fiberotomy enhances orthodontic tooth movement and diminishes relapse in a rat model. Orthod Craniofac Res 16, 161-168.

5. Kim SJ, Park YG, Kang SG (2009) Effects of Corticision on paradental remodeling in orthodontic tooth movement. Angle Orthod 79, 284-291.

6. Yen SL (2016) Comparison between osteotomy and corticotomy-assisted tooth movement. Front Oral Biol 18, 124-129.

7. Kanzaki H, Chiba M, Shimizu Y, Mitani H (2002) Periodontal ligament cells under mechanical stress induce osteoclastogenesis by receptor activator of nuclear factor kappaB ligand up-regulation via prostaglandin E2 synthesis. J Bone Miner Res 17, 210-220.

8. Kanzaki H, Chiba M, Arai K, Takahashi I, Haruyama N, Nishimura M et al. (2006) Local RANKL gene transfer to the periodontal tissue accelerates orthodontic tooth movement. Gene Ther 13, 678-685.

9. Yamasaki K, Shibata Y, Fukuhara T (1982) The effect of prostaglandins on experimental tooth movement in monkeys. J Dent Res 61, 1444-1446.

10. Soma S, Matsumoto S, Higuchi Y, Takano-Yamamoto T, Yamashita K, Kurisu K et al. (2000) Local and chronic application of PTH accelerates tooth movement in rats. J Dent Res 79, 1717-1724.

11. Showkatbakhsh R, Jamilian A, Showkatbakhsh M (2010) The effect of pulsed electromagnetic fields on the acceleration of tooth movement. World J Orthod 11, 52-56.

12. Yoshida T, Yamaguchi M, Utsunomiya T, Kato M, Arai Y, Kaneda $\mathrm{T}$ et al. (2009) Low-energy laser irradiation accelerates the velocity of tooth movement via stimulation of the alveolar bone remodeling. Orthod Craniofac Res 12, 289-298.

13. Genc G, Kocadereli I, Tasar F, Kilinc K, El S, Sarkarati B (2013) Effect of low-level laser therapy (LLLT) on orth- odontic tooth movement. Lasers Med Sci 28, 41-47.

14. Nishimura M, Chiba M, Ohashi T, Sato M, Shimizu Y, Igarashi $\mathrm{K}$ et al. (2008) Periodontal tissue activation by vibration: intermittent stimulation by resonance vibration accelerates experimental tooth movement in rats. Am J Orthod Dentofacial Orthop 133, 572-583.

15. Tsuka Y, Fujita T, Shirakura M, Kunimatsu R, Su SC, Fujii E et al. (2016) Effects of neodymium-doped yttrium aluminium garnet (Nd:YAG) laser irradiation on bone metabolism during tooth movement. J Lasers Med Sci 7, 40-44.

16. Ohshiro T, Calderhead RG (1991) Development of low reactive-level laser therapy and its present status. J Clin Laser Med Surg 9, 267-275.

17. Aleksic V, Aoki A, Iwasaki K, Takasaki AA, Wang CY, Abiko $Y$ et al. (2010) Low-level Er:YAG laser irradiation enhances osteoblast proliferation through activation of MAPK/ERK. Lasers Med Sci 25, 559-569.

18. Kawasaki K, Shimizu N (2000) Effects of low-energy laser irradiation on bone remodeling during experimental tooth movement in rats. Lasers Surg Med 26, 282-291.

19. Zhang W, Tang Y, Liu C, Shen Y, Feng X, Gu Y (2018) Root and root canal variations of the human maxillary and mandibular third molars in a Chinese population: a microcomputed tomographic study. Arch Oral Biol 95, 134-140.

20. Begg PR (1956) Differential force in orthodontic treatment. Am J Orthod 42, 481-510.

21. Ninomiya T, Miyamoto $Y$, Ito T, Yamashita A, Wakita M, Nishisaka T (2003) High-intensity pulsed laser irradiation accelerates bone formation in metaphyseal trabecular bone in rat femur. J Bone Miner Metab 21, 67-73.

22. Fujita S, Yamaguchi Y, Utsunomiya T, Yamamoto H, Kasai K (2008) Low-energy laser irradiation stimulates tooth movement velocity via expression of RANK and RANKL. Orthod Craniofac Res 11, 143-155.

23. Verschueren RC, Koudstaal J, Oldhoff J (1975) The carbon dioxide laser, some possibilities in surgery. Acta Chir Belg 74, 197-204.

24. Ogasawara T, Yoshimine Y, Kiyoshima T, Kobayashi I, Matsuo K, Akamine A et al. (2004) In situ expression of RANKL, RANK, osteoprotegerin and cytokines in osteoclasts of rat periodontal tissue. J Periodontal Res 39, 42-49. 